\title{
10 years later...
}

\author{
Louis Valiquette MD MSc FRCPC ${ }^{1}$, Kevin B Laupland MD MSc FRCPC 2,3
}

$\mathrm{O}^{\mathrm{n}}$ November 16, 2002, in the city of Foshan in the province of Guangdong (China), a 45-year-old man developed a severe atypical pneumonia and further infected four relatives. This was the first case of severe acute respiratory syndrome (SARS), as demonstrated by epidemiological investigations (1). Almost exactly 10 years later, on November 8, 2012, the first report of a mortal case of acute pneumonia associated with renal failure due to a novel coronavirus in a businessman from Bisha, Saudi Arabia, was published (2). In retrospect, we now know that the first confirmed human cases arose in a cluster of 13 individuals with respiratory symptoms in a hospital in Zarqa, Jordan, in April 2012 (3,4).

Middle East respiratory syndrome coronavirus (MERS-CoV) belongs to the Coronaviridae family, a group of large, enveloped, singlestranded RNA viruses. Similar to most RNA viruses, they are known for their genomic plasticity, and infect mammalian and avian hosts. It is the fourth identified beta coronavirus with the ability to infect humans (the others are SARS-CoV, human CoV-HKU1 and human $\mathrm{CoV}$-OC43). It belongs to lineage $\mathrm{C}$, shares similarities with viruses identified in the Pipistrellus species of bats (HKU4 and HKU5) and has been demonstrated to replicate in various bat cell lines (4). Consequently, a bat origin for MERS-CoV has been suggested. However, no clear and repetitive exposure to bats has been reported in epidemiological studies, and the search for an intermediate host has led researchers to identify dromedary camels as potential suspects (5). The identification of MERS-CoV-neutralizing antibodies in camels from Oman and the Canary Islands, where no human case has been reported, raises more questions than answers.

It must be recognized that new information is being reported constantly and that the data presented in the present report may soon be outdated. As of October 18, 2013, 139 laboratory-confirmed cases of MERS-CoV have been reported, including 60 deaths, representing a case fatality rate of $43 \%$. All cases have been directly or indirectly linked to travel or residence in four countries: Saudi Arabia, Qatar, Jordan and the United Arab Emirates (6).

According to the WHO, a case is 'probable' when it combines the three following clinical, epidemiological and laboratory criteria (7):

- Febrile illness + evidence of parenchymal pulmonary disease (pneumonia or acute respiratory distress syndrome) AND testing for MERS-CoV is unavailable or negative on a single inadequate specimen AND there is a direct epidemiological link with a confirmed case;

- Febrile illness + evidence of parenchymal pulmonary disease (pneumonia or acute respiratory distress syndrome) AND presence of a positive screening test without confirmation (inconclusive) AND resident or traveller to Middle Eastern countries where MERS-CoV was circulating within 14 days of onset;

- Acute febrile respiratory illness of any severity AND presence of a positive screening test without confirmation (inconclusive) AND direct epidemiological link with a confirmed case.

A case is confirmed when a patient has laboratory documentation of MERS-CoV infection. A testing algorithm for cases under investigation using real-time reverse transcriptase polymerase chain reaction was published in September 2013 (8). For the moment, cases cannot be confirmed by serological testing only, even if highly specific assays have been described, because they have been validated on a limited number of patients (9-11). A case with a positive serological test in the absence of polymerase chain reaction/sequencing confirmation is, therefore, considered to be 'probable'. It is important to mention that one cannot reliably rule out MERS-CoV on a single negative result because virus shedding (similar to SARS) in the respiratory tract varies according to the specimen and the time since onset of illness (11).

The incubation period of MERS-CoV was measured using data from a well-defined hospital outbreak in Al-Hasa in Eastern Saudi Arabia, and estimated to be 5.2 days (95\% CI 2.2 to 12.4 days) (12). This is similar to SARS-CoV, which has an average incubation period of four days (95\% CI 1.8 to 10.6 days) (13). The serial interval time is the period of time between analogous phases of an infectious illness in successive cases of a chain of infection that is spread from person to person. In the same report, this was defined as 7.6 days for MERS-CoV (versus 8.4 days for SARS) $(12,14)$.

Well-described clusters have been published. Despite several close family contacts with index cases, only a small number of secondary cases have been identified $(12,15)$. The reproduction number $\left(R_{0}\right)$, defined as the number of cases generated by one infected individual on average in a fully susceptible population, is a very important epidemiological measure. When $R_{0}$ is $<1$, the infection will usually die out over time, and when $R_{0}$ is $>1$, there is a clear risk of spreading in the population. Breban et al (16) calculated the MERS-CoV $\mathrm{R}_{0}$ to be 0.69 (95\% CI 0.5 to 0.92 ) in the worst-case scenario. Comparatively, they estimated the $\mathrm{R}_{0}$ for SARS during the prepandemic stage in Southern Asia to be 0.80 (95\% CI 0.54 to 1.13). It is expected that $R_{0}$ will be updated as more data are reported because it may change seasonally according to school calendars and yearly gatherings, such as the Hajj pilgrimage, and if the virus evolves. Recent molecular data support the hypothesis of multiple introductions to explain the origin of cases linked in the same hospital outbreak (17). These data have a clear impact on $\mathrm{R}_{0}$ estimation, which will probably revise $\mathrm{R}_{0}$ downward.

At this time, the main mechanism of transmission is not well defined. In some reports, secondarily infected patients were $>1 \mathrm{~m}$ from the index case, which supports potential airborne transmission (12). For the moment, standard, contact and airborne precautions are recommended by the Centers for Disease Control and Prevention (Georgia, USA) and by Health Canada for health care workers involved in the management of suspected MERS-CoV cases or close contacts of confirmed cases (within 14 days of onset). These recommendations are consistent with those of SARS and were influenced by the high rate of morbidity and mortality in infected patients, unknown modes of transmission, potential human-tohuman transmission, and lack of vaccine or chemoprophylaxis $(18,19)$.

Assiri et al (20) recently reported a clinical comparison between patients infected with MERS-CoV and those infected with SARS. It is important to specify that this analysis is preliminary because it was performed on a limited number of subjects $(n=47)$ and, consequently, is subject to change. General data were updated from other sources (Table 1). As with preliminary reports of SARS patients, data from cohorts described initially may strongly differ from larger, later cohorts. With SARS, the initial published cohorts included several health care workers $(>50 \%)$ who were younger and healthier than the hospital

${ }^{1}$ Department of Microbiology-Infectious Diseases, Université de Sherbrooke, Sherbrooke, Quebec; ${ }^{2}$ Departments of Medicine, Critical Care Medicine, Pathology and Laboratory Medicine and Community Health Sciences, University of Calgary, Calgary, Alberta; ${ }^{3}$ Department of Medicine, Royal Inland Hospital, Kamloops, British Columbia

Correspondence: Dr Louis Valiquette, Université de Sherbrooke, 3001 12ème Avenue Nord, Sherbrooke, Quebec J1H 5 N4. Telephone 819-346-1110 ext 12568,fax 819-829-3289,e-mail louis.valiquette@usherbrooke.ca 


\begin{tabular}{|c|c|c|}
\hline Characteristic & MERS-CoV & SARS \\
\hline \multicolumn{3}{|l|}{ Demographic data } \\
\hline$\geq 18$ years of age ${ }^{*}$ & 98 & 93 \\
\hline Median age (range), years ${ }^{\dagger}$ & $50(2-94)$ & $40(1-91)$ \\
\hline Male:female ratio $(n=130)^{\dagger}$ & $1.6: 1$ & $1: 1.3$ \\
\hline \multicolumn{3}{|l|}{ Outcomes and clinical features } \\
\hline Case fatality rate $\ddagger$ & $\mathrm{n}=60 / 139(43 \%)$ & $0-40$ \\
\hline Time from onset to death, days* & 16.5 & 23.7 \\
\hline \multicolumn{3}{|l|}{ Symptoms* } \\
\hline Fever & 98 & $99-100$ \\
\hline Cough & 83 & $62-100$ \\
\hline Hemoptysis & 17 & $0-1$ \\
\hline Shortness of breath & 72 & $40-42$ \\
\hline Vomiting & 21 & $20-35$ \\
\hline Diarrhea & 26 & $20-25$ \\
\hline \multicolumn{3}{|l|}{ Comorbidities* } \\
\hline Any & 96 & $10-30$ \\
\hline Diabetes & 68 & 24 \\
\hline Chronic renal failure & 49 & $2-6$ \\
\hline Chronic heart disease & 28 & 10 \\
\hline \multicolumn{3}{|l|}{ Laboratory and imaging } \\
\hline Chest x-ray abnormalities* & 100 & $94-100$ \\
\hline Leukopenia $\left(<4.0 \times 10^{9} \mathrm{cells} / \mathrm{L}\right)^{*}$ & 14 & $25-35$ \\
\hline Lymphopenia $\left(<1.5 \times 10^{9} \text { cells } / \mathrm{L}\right)^{*}$ & 34 & $68-85$ \\
\hline $\begin{array}{l}\text { Increased alanine aminotransferase/ } \\
\text { aspartate aminotransferase level }{ }^{*}\end{array}$ & $11 / 15$ & $20-30 / 20-30$ \\
\hline
\end{tabular}

Data presented as \% unless otherwise indicated. *Data adapted from Assiri et

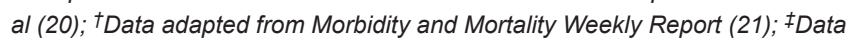
adapted from $\mathrm{WHO}$ (6)

population. With MERS-CoV, several cases in early reports included patients attending the hemodialysis unit. Ongoing evaluation is required to assess whether chronic renal disease is a true risk factor for the development of MERS-CoV infection.

Although there is an ever-increasing body of evidence being reported, there are many knowledge gaps that need to be addressed (20). The origin and the natural reservoir for MERS-CoV remain obscure. The overall incidence and risk factors for acquisition of symptomatic disease and adverse outcome are not well defined. Our limited understanding of transmissibility of this pathogen challenges our ability to define optimal infection prevention and control procedures. We do not know whether there are any specific therapies beyond supportive treatments that will improve outcome.

Only the future will define whether MERS-CoV will be a limited regional phenomenon or whether a pandemic will ensue. While we cannot be certain of the future of MERS-CoV, we believe that the next decade(s) will inevitably witness the emergence of other novel respiratory viruses that, along with SARS and MERS-CoV, will have a profound influence on the global community.

\section{REFERENCES}

1. Hilgenfeld R, Peiris M. From SARS to MERS: 10 years of research on highly pathogenic human coronaviruses. Antiviral Res 2013;100:286-95.

2. Zaki AM, van Boheemen S, Bestebroer TM, Osterhaus AD, Fouchier RA. Isolation of a novel coronavirus from a man with pneumonia in Saudi Arabia. N Engl J Med 2012;367:1814-20.
3. European Centre For Disease Prevention and Control (ECDC). Severe respiratory disease of unknown origin - Jordan - outbreak in ICU. 2012. < http://www.ecdc.europa.eu/en/publications/ Publications/CDTR\%20online\%20version\%204\%20May\%202012. pdf $>$ (Accessed October 18, 2013).

4. de Groot RJ, Baker SC, Baric RS, et al. Middle East respiratory syndrome coronavirus (MERS-CoV): Announcement of the Coronavirus Study Group. J Virol 2013;87:7790-2.

5. Reusken CB, Haagmans BL, Muller MA, et al. Middle East respiratory syndrome coronavirus neutralising serum antibodies in dromedary camels: A comparative serological study. Lancet Infect Dis 2013;13:859-66.

6. World Health Organization (WHO). Global Alert and Response (GAR): Novel coronavirus infection. 2013. <www.who.int/csr/ don/2013_10_18/en/index.html> (Accessed October 18, 2013).

7. World Health Organization (WHO). Revised interim case definition for reporting to $\mathrm{WHO}$ - Middle East respiratory syndrome coronavirus (MERS-CoV). 2013. <www.who.int/csr/ disease/coronavirus_infections/case_definition/en/> (Accessed October 18, 2013).

8. World Health Organization. Laboratory Testing for Middle East Respiratory Syndrome Coronavirus Interim recommendations. 2013. <www.who.int/csr/disease/coronavirus_infections/MERS_ Lab_recos_16_Sept_2013.pdf> (Accessed October 18, 2013).

9. Buchholz U, Muller MA, Nitsche A, et al. Contact investigation of a case of human novel coronavirus infection treated in a German hospital, October - November 2012. Euro Surveill 2013;18:20406.

10. Reusken C, Mou H, Godeke GJ, et al. Specific serology for emerging human coronaviruses by protein microarray. Euro Surveill 2013;18:20441.

11. Drosten C, Seilmaier M, Corman VM, et al. Clinical features and virological analysis of a case of Middle East respiratory syndrome coronavirus infection. Lancet Infect Dis 2013;13:745-51.

12. Assiri A, McGeer A, Perl TM, et al. Hospital outbreak of Middle East respiratory syndrome coronavirus. N Engl J Med 2013;369:407-16.

13. Lessler J, Reich NG, Brookmeyer R, Perl TM, Nelson KE, Cummings DA. Incubation periods of acute respiratory viral infections: A systematic review. Lancet Infect Dis 2009;9:291-300.

14. Lipsitch M, Cohen T, Cooper B, et al. Transmission dynamics and control of severe acute respiratory syndrome. Science 2003;300:1966-70.

15. Memish ZA, Zumla AI, Al-Hakeem RF, Al-Rabeeah AA, Stephens GM. Family cluster of Middle East respiratory syndrome coronavirus infections. N Engl J Med 2013;368:2487-94.

16. Breban R, Riou J, Fontanet A. Interhuman transmissibility of Middle East respiratory syndrome coronavirus: Estimation of pandemic risk. Lancet 2013;382:694-9.

17. Cotten M, Watson SJ, Kellam P, et al. Transmission and evolution of the Middle East respiratory syndrome coronavirus in Saudi Arabia: A descriptive genomic study. Lancet 2013; September 24 (Epub ahead of print).

18. Centers for Disease Control and Prevention. Interim Infection Prevention and Control Recommendations for Hospitalized Patients with Middle East Respiratory Syndrome Coronavirus (MERS-CoV). 2013. <www.cdc.gov/coronavirus/mers/infection-prevention-control. html > (Accessed October 18, 2013).

19. Public Health Agency of Canada. Interim Guidance - Middle East respiratory syndrome coronavirus (MERS-CoV). 2013. <www.phacaspc.gc.ca/eri-ire/coronavirus/guidance-directives/nCoV-ig-dp-eng. php> (Accessed October 18, 2013).

20. Assiri A, Al-Tawfiq JA, Al-Rabeeah AA, et al. Epidemiological, demographic, and clinical characteristics of 47 cases of Middle East respiratory syndrome coronavirus disease from Saudi Arabia: A descriptive study. Lancet Infect Dis 2013;13:752-61.

21. Updated information on the epidemiology of Middle East respiratory syndrome coronavirus (MERS-CoV) infection and guidance for the public, vlinicians, and public health authorities, 2012-2013. MMWR Morb Mortal Wkly Rep 2013;62:793-6. 


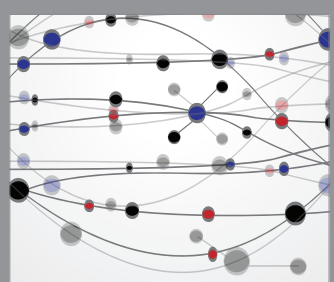

The Scientific World Journal
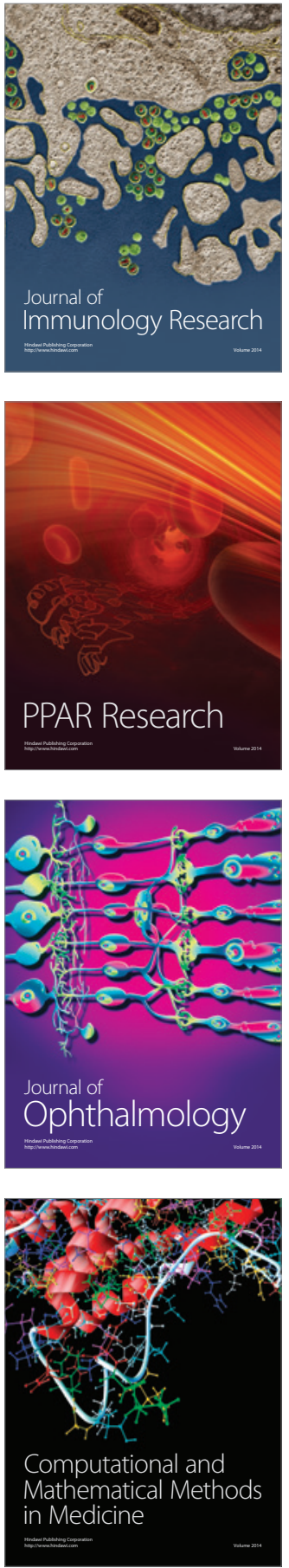

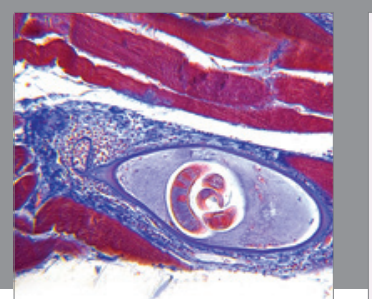

Gastroenterology Research and Practice

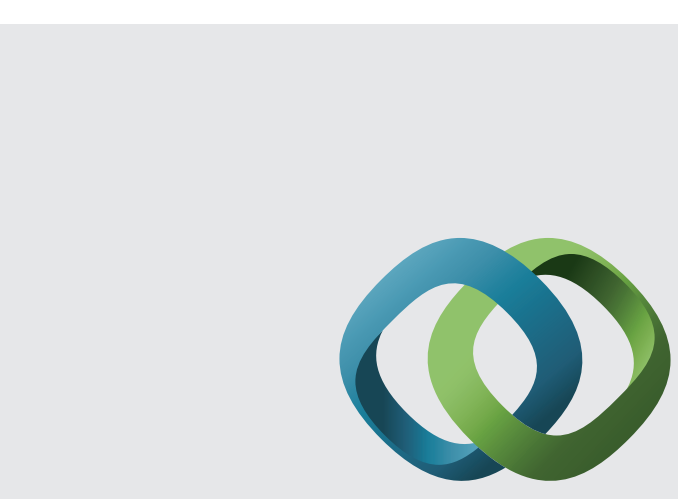

\section{Hindawi}

Submit your manuscripts at

http://www.hindawi.com
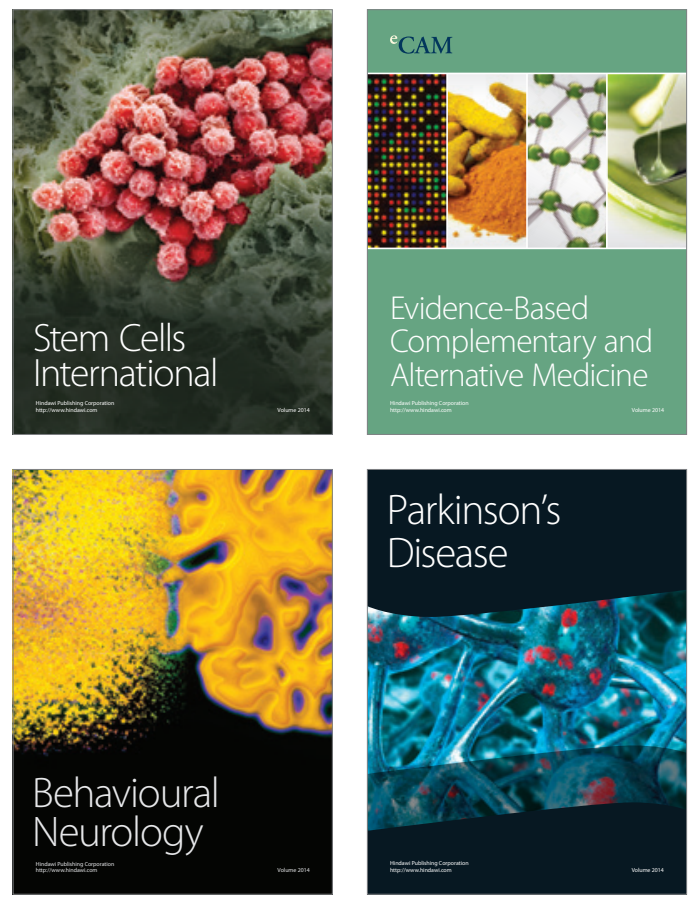
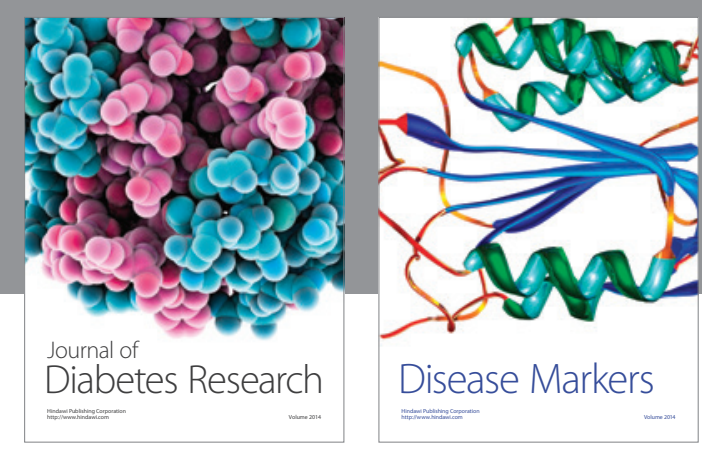

Disease Markers
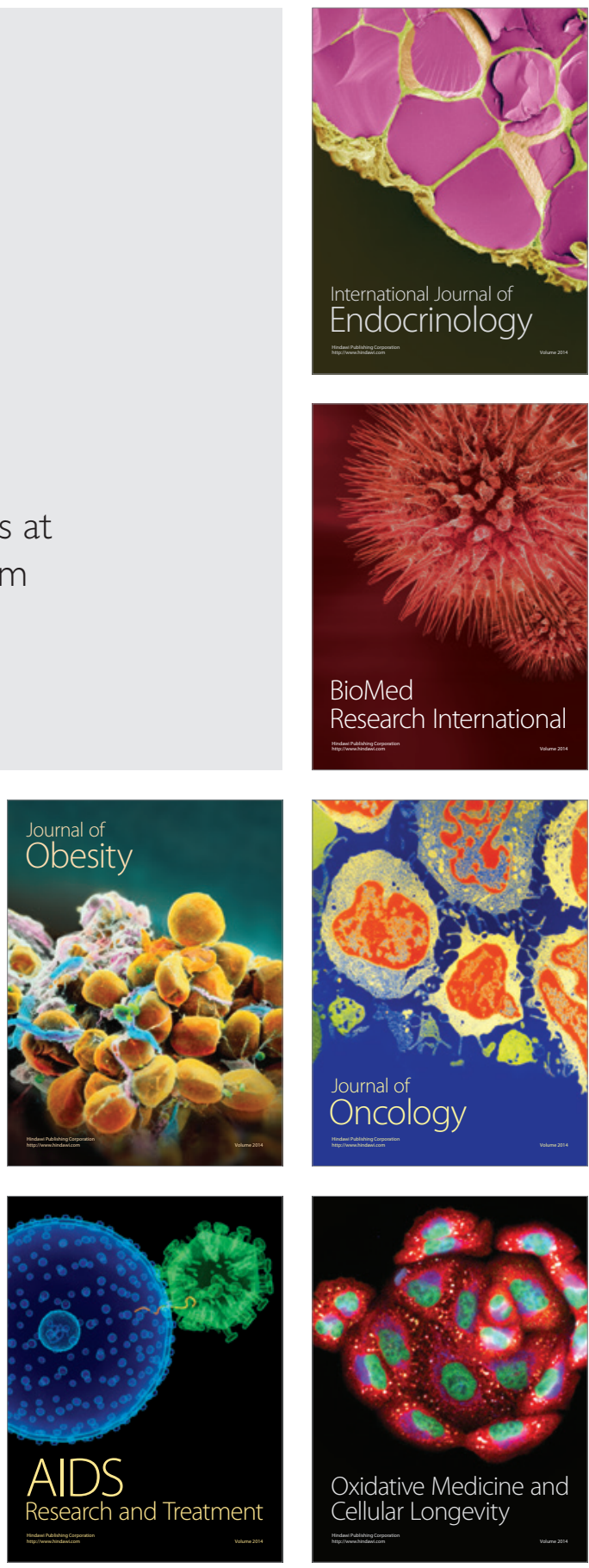